\title{
An Alternative Inverse S-Transform for Filters with Time-Frequency Localization
}

\author{
M. Schimmel and J. Gallart \\ Institute of Earth Sciences Jaume Almera - CSIC \\ c/ Lluis Sole i Sabaris, s/n, \\ 08028 Barcelona, Spain. \\ schimmel@ija.csic.es
}

\author{
C. Simon \\ Marine Technology Unit - CSIC \\ Passeig Maritim de la Barceloneta, 37 \\ 08003 Barcelona, Spain. \\ csimon@utm.csic.es
}

\begin{abstract}
The S-Transform provides a framework for dataadaptive filters which take advantage of time-frequency localized spectra. These filters basically consist in a data transformation to the time-frequency domain, a dataadaptive weighting of the localized spectra, and a back transformation. We illustrate that the inverse S-Transform of manipulated spectra not necessarily transforms the localized signals as expected from the imposed weighting. The time localization is not directly translated and spurious signals and noise can be generated. We discuss this problem and suggest a new inverse S-Transform which may come helpful to many applications to take more advantage of the time-frequency localization.
\end{abstract}

\section{Introduction}

The S-Transform by Stockwell, Mansinha and Lowe [1] can be seen as an extension of the short-time Fourier Transform which uses frequency dependent scaling windows in analogy to the wavelet transform. This method permits to transform a time series into the time-frequency domain using a frequency dependent resolution with narrower windows at higher frequencies and wider windows at lower frequencies. It is a powerful tool which has been used in several motivating studies such as medical imaging, acoustics, electrical engineering, geophysics, among others. Most studies use Gaussian shaped windows, but other windows can also be employed ([2], [3]).

The time-frequency representation obtained with the STransform is unique and invertible. When averaged over time, the S-Transform becomes the Fourier Transform of the original time series. This is used to invert S-Transforms and permits to transform freely between the time, frequency and time-frequency domain. The simultaneous localization in time and frequency often permits signal isolation and filters can be constructed to operate on the S-spectra before their back transformation to time. These filters consist in the data adaptive weighting of the spectral components due to a measure which separates to some extend signals from noise (e.g., degree of polarization, coherence, or energy). The higher weights localize regions in the time-frequency spectrum which are expected to be the signal components while the lower weights attenuate undesired noise. The inverse S-transform is computed to reconstruct the filtered time series from the weighted $\mathrm{S}$-spectrum. This procedure can be employed to remove motion-related artefacts from medical magnetic resonance images, unpolarized and/or incoherent signals (noise) from seismic record sections, among others.

It has been shown in [5] that the inverse S-Transform of manipulated S-spectra not necessarily transforms the localized signals as expected from the imposed weighting. The time localization is not directly translated and spurious signals and noise can be generated. Here, we closely follow [5] to discuss this problem and to present the proposed inverse S-Transform which may come helpful to many applications to take more advantage of the time-frequency localization.

\section{Time-frequency localization with S- transform}

\subsection{Localized spectra determination with gener- alized S-transform}

The S-Transform of a time series $u(t)$ is following Stockwell et al. [1]

$$
S(\tau, f)=\int_{-\infty}^{\infty} u(t) w(\tau-t, f) e^{-i 2 \pi f t} d t
$$

with Gaussian window

$$
w(\tau-t, f)=\frac{|f|}{k \sqrt{2 \pi}} e^{\frac{-f^{2}(\tau-t)^{2}}{2 k^{2}}}, \quad k>0 .
$$

$f$ is frequency and $\tau, t$ are time variables. $\tau$ is the center time of the Gaussian window and $k$ is a scaling factor which controls the number of oscillations in the window. $k$ permits to control the time-frequency resolution. A high precision in both, time and frequency, can not be obtained due to the uncertainty principle. 


\subsection{Inverse transformation of S-spectra}

\subsubsection{Routinely used approach}

The local time-frequency spectra $S(\tau, f)$ obtained with the generalized S-Transform can easily be back-transformed (e.g., [1], [3]) since the S-transform windows satisfy the condition

$$
\int_{-\infty}^{\infty} w(\tau-t, f) d \tau=1
$$

This ensures that the time averaging of the S-spectrum $S(\tau, f)$ yields the spectrum $U(f)=F T[u(t)]$ as shown in equation (4).

$$
\int_{-\infty}^{\infty} S(\tau, f) d \tau=\int_{-\infty}^{\infty} u(t) e^{-i 2 \pi f t} \int_{-\infty}^{\infty} w(\tau-t, f) d \tau d t=U(f)
$$

It means that the S-transform is exactly invertible with one inverse Fourier Transform. This permits a fast and easy retrieval of the time series from their S-spectrum. If the spectrum is manipulated then the back transformation provides a filtered time series. A manipulation of the spectrum can be the weighting of the different spectral components based on criteria such as coherence, polarization, energy, etc. to enhance/attenuate certain features in the data. This procedure permits to eliminate undesired parts of the data in the time-frequency domain. Equation (5) expresses this filter strategy with $F(\tau, f)$ as a data-adaptive time-frequency weighting function.

$$
u_{f i l t_{1}}(t)=\int_{-\infty}^{\infty} \int_{-\infty}^{\infty} S(\tau, f) F(\tau, f) e^{+i 2 \pi f t} d \tau d f
$$

$F(\tau, f)$ is real, any complex function would manipulate the phase spectrum.

\subsubsection{New approach}

A second concept has been proposed in [5]. They derived the following back transformation (equation 6) from a timetime representation of the windowed time series after recognizing a relation with the $\mathrm{S}$-spectrum.

$$
u(t)=k \sqrt{2 \pi} \int_{-\infty}^{\infty} \frac{S(t, f)}{|f|} e^{+i 2 \pi f t} d f
$$

The filtered trace, in analogy to $u_{f i l t_{1}}(t)$, is expressed as

$$
u_{f i t_{2}}(t)=k \sqrt{2 \pi} \int_{-\infty}^{\infty} \frac{S(t, f) F(t, f)}{|f|} e^{+i 2 \pi f t} d f
$$

In contrast to equation (5) the phasor is assigned to the time variable of the S-spectrum and its weight, and therefore conserves the time localization imposed by the weight. This time localization has been lost in the summation over $\tau$ and the inverse Fourier transform in equation (5). Moreover, the factor $k \sqrt{2 \pi} /|f|$ cancels with the frequency dependent amplitude of the Gauss window $w(\tau-t, f)$ used to determine $S(t, f)$.

\subsection{Differences for manipulated S-spectra}

The differences between both strategies (equations 5 and 7) can be shown with a hypothetical filter $F\left(t=t_{1}, f\right)=1$ and $F\left(t \neq t_{1}, f\right)=0$ elsewhere. That is, at time $t=$ $t_{1}$ all frequency components of the S-spectrum are passed while at all other times and frequencies the S-spectrum is suppressed by $F(t, f)$. Employing this operation, equation (5) reduces to

$$
u_{f i l t_{1}}(t)=\int_{-\infty}^{\infty} S\left(t_{1}, f\right) e^{+i 2 \pi f t} d f
$$

Each frequency component $S\left(t_{1}, f\right)$ is transformed to a sine wave which together may or may not cancel at $t \neq t_{1}$. One observes that the zero weight of $F(t, f)$ at $t \neq t_{1}$ does not directly map into the time domain. With the second approach (equation 7) one obtains

$$
\begin{gathered}
u_{f i l t_{2}}\left(t_{1}\right)=\int_{-\infty}^{\infty} \frac{k \sqrt{2 \pi}}{|f|} S\left(t_{1}, f\right) e^{+i 2 \pi f t_{1}} d f \\
\text { and } u_{\text {filt }}\left(t \neq t_{1}\right)=0
\end{gathered}
$$

which shows that the time localization through the $F(t, f)$ translates directly into the time domain.

Figure 1 illustrates this in an extended example. The test trace $u(t)$ is a $2-\mathrm{Hz}$ sine wave as depicted in Figure 1a. The time frequency representation in Figure 1b shows the amplitudes of the S-spectrum. (Note that $k=1$ is used throughout all examples.) In the next step the S-spectrum is manipulated by multiplying all spectral components in the white area (Figure 1b) by zero. The manipulated spectrum is then back transformed to the time domain using both concepts (Figure 1c). It is visible from the filtered traces that only with the second strategy the imposed time localization translates directly to the time domain in analogy to equation (9).

For a further test we add three sine functions of $0.3,2$, and $3.5 \mathrm{~Hz}$ frequencies (Figure 1d). Its $\mathrm{S}$-spectrum has been manipulated similarly to previous example. However, we use a time duration of $5 \mathrm{~s}$ and $30 \mathrm{~s}$ for the pass-band (multiplication by 1) and show the filtered signals in Figures $1 \mathrm{e}$ and $1 \mathrm{f}$, respectively. The examples demonstrate that there are no zero amplitude stretches as one would expect from the imposed S-spectrum manipulation, when applying the first inversion strategy. Instead, one observes due to linearity the superposition of sine waves, each generated as in equation (8). The zero amplitude weights are not sufficient to guarantee a cancellation of the sine waves in the time domain.

Note that the S-transform of none of the filtered traces would reproduce the manipulated S-spectrum. The Sspectrum of the filter output of the first approach has energy at the frequencies of the sine functions at all times. The 

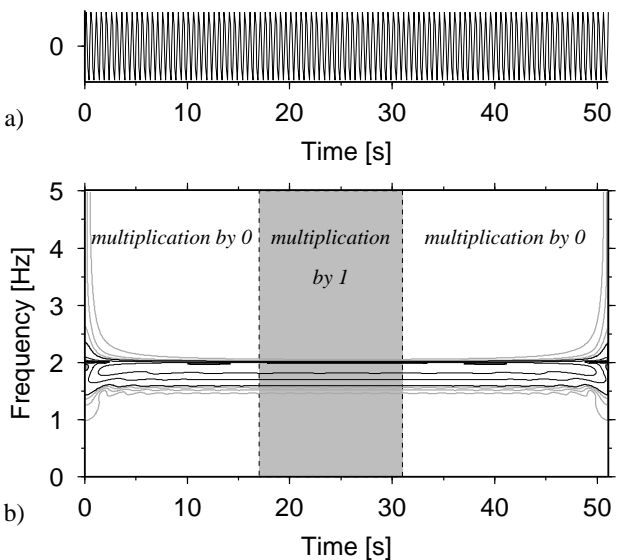

0

d) 0
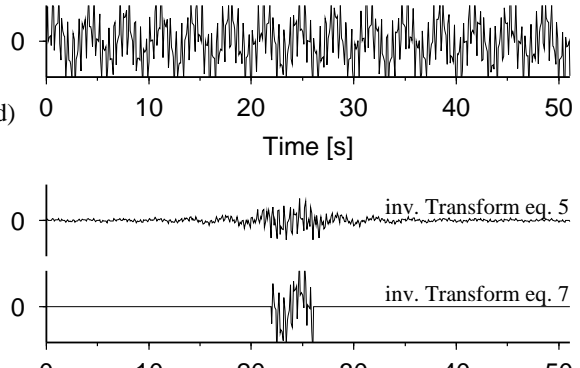

e) 0

1020

[s]
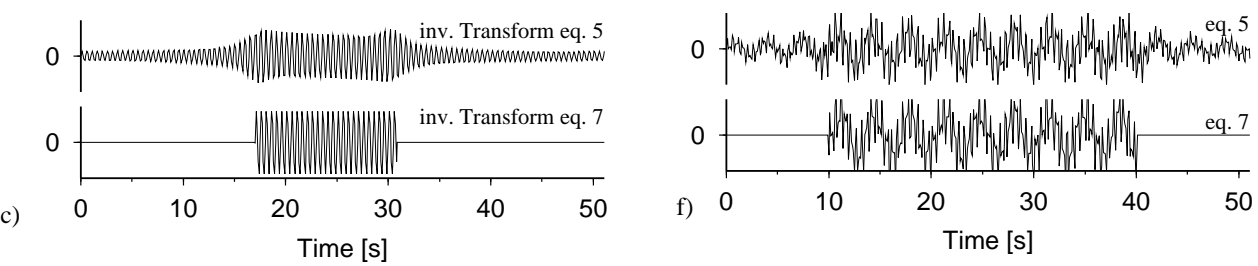

Figure 1. a) shows a 2-Hz sine wave and b) its amplitude S-spectrum. The black lines have a contour interval of $20 \%$ of the maximum amplitude and the grey lines contour the lower $20 \%$ with a $5 \%$ increment. The shaded background indicates the S-spectrum manipulation. c) Results of the back transformation of the manipulated S-spectrum. d) A second test trace constructed from three sine functions with $0.3,2$, and $3.5 \mathrm{~Hz}$ frequencies. The corresponding S-spectrum has been manipulated similar to b), however, with a $5 \mathrm{~s}$ and $\mathbf{3 0} \mathrm{s}$ time duration of the band-pass. The back transformed time series are shown in e) and f), respectively.

S-spectrum of the filtered trace with the second approach has smeared amplitudes at the begin and end of the nonzero amplitude signal. We therefore understand the back transform of the manipulated spectrum as part of the filter operation.

More examples can be found in [5] and will be discussed at the ISPA 2005 Symposium in Zagreb.

\subsection{Examples with data-adaptive polarization fil- ter}

Previous case study demonstrate that the filter efficiency depends on the inverse transform. Data and manipulation have been constructed to point to these differences. Now we consider an authentic data-adaptive filter which we apply to theoretical data and a real data set to illustrate differences and importance for a realistic situation.

We use three-component (3-c) data and apply a polarization filter to enhance arbitrarily polarized signals in the data. In essence, the filter consists in the determination of a frequency dependent degree of polarization (dop) which we use to weight the S-spectra. The dop is taken from Schimmel and Gallart [4]. It is an instantaneous measure on how well a signal is polarized based on the stability of its arbitrary polarization within a small sliding data window. It assigns at each time and frequency a real number between 0 and 1 which is used to weight the components of the S-spectrum in analogy to $F(t, f)$ in equations (5) and (7). Components with a low dop are down weighted to enhance/isolate the polarized signal with a high dop.

In the first example (Figure 2) we employ a theoretical 3 -c registration of a polarized signal. The noise free signal on the vertical $(\mathrm{Z})$, radial $(\mathrm{R})$ and transverse $(\mathrm{T})$ component can be seen in the first line of Figure 2. We add some white noise to contaminate the polarized signal and to obtain our filter input which is shown in the second line of Figure 2. The third and fourth lines contain the polarization filter outputs with the routine inverse transform (equation 5) and the new inverse transform (equation 7). The overall noise has been attenuated with both approaches, however, the filter output obtained with equation (7) mostly resembles to the noise-free test signal. Some of the small energy features belong to the background noise which occasionally can be polarized. This can be revealed by inspection of the dop matrix which mask and isolates signals from noise through the attenuation of less polarized signals. It can be seen that the potential of the filter is best exploited employing the second approach as inverse transform.

In the following we use 3-c seismic recordings of an earthquake with epicenter at Primor in Russia (2003 July $27,47^{\circ} \mathrm{N}, 139^{\circ} \mathrm{E}, 470 \mathrm{~km}$ depth, $m_{b}=6.3$ ) recorded at 10 mobile broad-band stations in NW Spain. Figure 3a shows the corresponding $\mathrm{Z}$ components after band passing (0.03 $1 \mathrm{~Hz}$ ). The labels and lines indicate the expected arrivals 

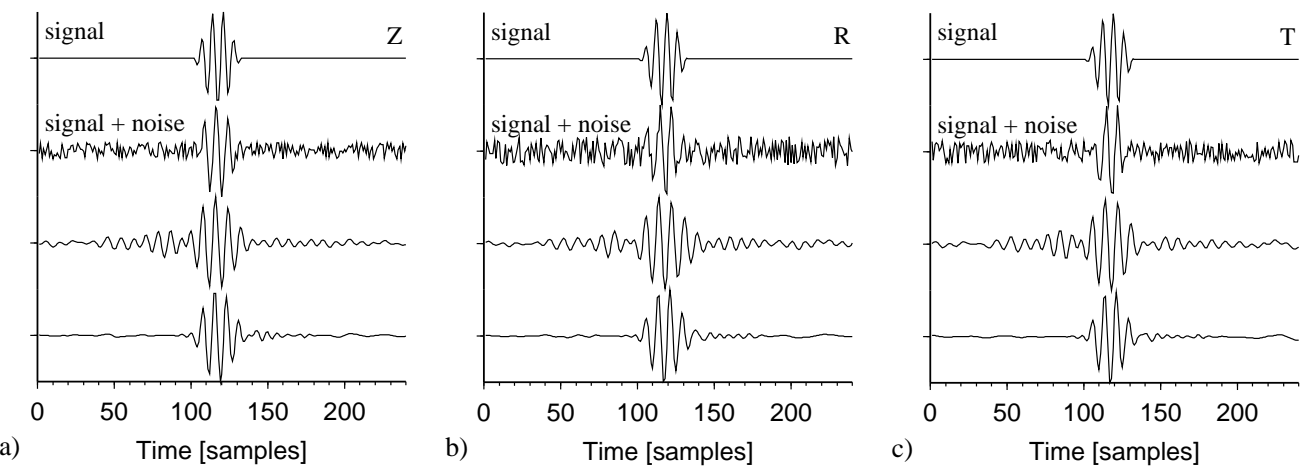

Figure 2. Theoretical three-component registration of a polarized signal. a) From top to bottom: noise-free signal on the vertical (Z) component, noise contaminated signal on Z, polarization filtered trace employing equation (5), polarization filtered trace employing equation (7). b) Same as a) but for the radial (R) component. c) Same as a) but for the transverse (T) component.
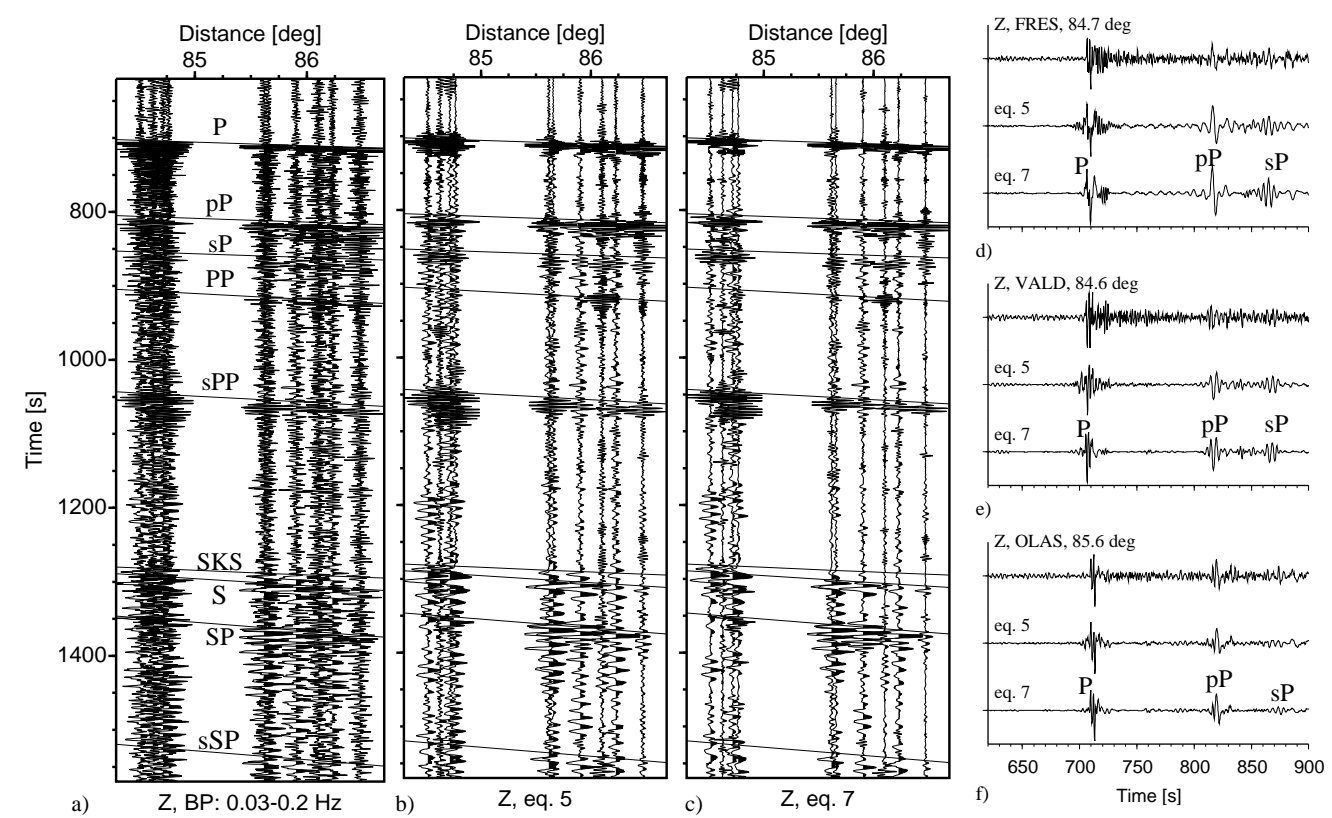

Figure 3. a) $Z$ component seismograms for an earthquake at Primor in Russia (2003 July 27) recorded at 10 mobile stations in NW Spain. Data are band-passed $(0.03-1 \mathrm{~Hz})$ and the labels indicate some main seismic signals. b) Polarization filtered record section. The approach, equation (5), is used for the inverse transform to the time domain. c) Same as b) but employing equation (7). d)-f) display a data zoom to enable inspection of the wave forms at three selected stations. 
of some prominent seismic phases. The first seismic wave from the Earthquake arrives as the $\mathrm{P}$ phase at about 700 s. The other phases are reverberations or wave type conversions at the Earth surface. The $\mathrm{P}$-wave and S-wave coda consists of many other reverberations and wave type conversions at the different structural discontinuities in the Earth. The signals before $700 \mathrm{~s}$ provide an estimate of the background noise in the data. Several seismic arrivals are already visible in the band-passed data (Figure 3a).

We apply the mentioned polarization filter to enhance the polarized signals. The data adaptive filter is used in its simplest form on the individual traces, i.e., without any lateral averaging of the dop or the waveforms to aid the attenuation of incoherent noise. The lateral averaging would further enhance coherent polarized signals through the attenuation of isolated polarized features which likely are polarized noise.

Figures $3 \mathrm{~b}$ and $3 \mathrm{c}$ show the filtered $\mathrm{Z}$ components. An overall noise suppression is visible and several signals have been enhanced. Further, it seems that the polarized signals lack the higher frequency components at increasing time. This is mainly attributed to attenuation due to scattering and inelasticity and the increased appearance of shear waves (Swaves) at larger time. Further, isolated polarized signals are attributed to polarized noise which can be due to some other local event/happening which is recorded. Seismic waves scattered at local heterogeneities can also be polarized and are often classified as signal generated noise.

The differences between Figures $3 b$ and $3 c$ are caused by the different approaches to transform the manipulated $\mathrm{S}$ spectra to time. Figures $3 \mathrm{~d}-\mathrm{f}$ are zooms of the filter input and outputs for three of the stations. It is visible that the noise level is smaller with equation (7) than with equation (5). Part of the signals in Figure $3 b$ (second traces in Figures $3 \mathrm{~d}-\mathrm{f}$ ) is introduced by the transform. Both approaches have been tested with data from several earthquakes and different filter settings. It is observed that differences between both transformations can be larger than shown in Figure 3.

The amplitude S-spectra of the traces for station OLAS (Figure 3d) are displayed in Figure 4a-c. The first arrival $\mathrm{P}$ and the event-site surface reflections $\mathrm{pP}$ and $\mathrm{sP}$ are visible in the three amplitude spectra. Figures $4 \mathrm{~b}$ and $4 \mathrm{c}$ are cleaned images of the input data due to the applied polarization filter. In Figure $4 c$ the signals appear more distinguished. This observation can be more or less pronounced for other data. The problem with real data is that it is not exactly known what is signal (and what is noise). This complication can obscure the filter performance and synthetic simulations become important for the interpretation.

\section{Discussion and conclusion}

We propose in [5] a new strategy for the inverse Stransform. This is shown to be relevant when the data processing involves a manipulation of the S-spectrum for feature extraction or noise attenuation. Our approach works differently to the routine inverse S-transform by Stockwell et al. [1]. Inherent to the differences, both approaches pro-
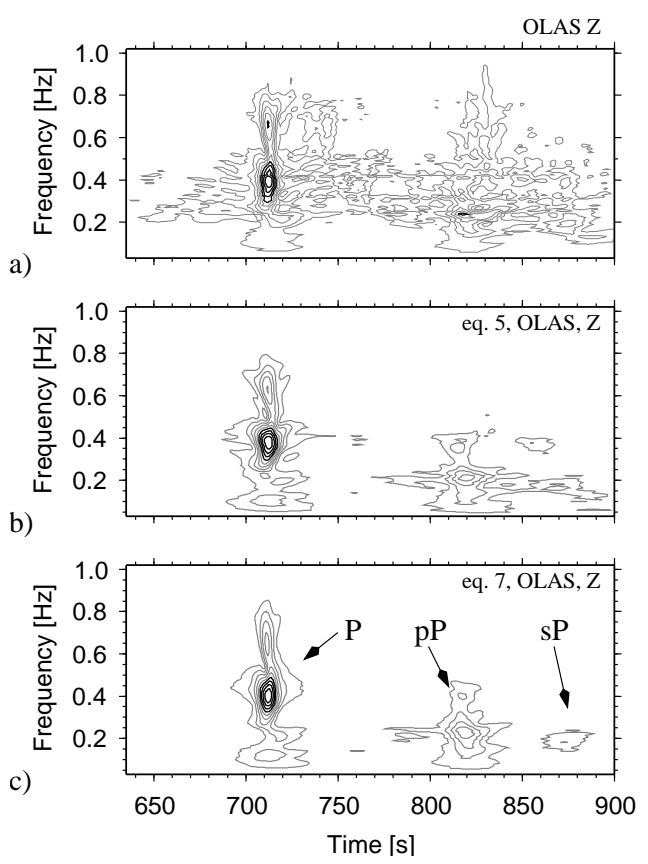

Figure 4. a) The amplitude S-spectrum of the $Z$ record of station OLAS (first trace in Figure 3f). The amplitudes are normalized to 1 and the contour interval is $\mathbf{0 . 2}$. The black lines indicate amplitudes larger than 0.6. b) Same as a) but for the filter output obtained with inverse transform eq. (5) (second trace in Figure 3f). c) Same as b) but using eq. (7) (third trace in Figure 3f).

vide different results whenever the spectrum is altered.

With the approach by Stockwell et al. [1] the S-spectrum is averaged over time to obtain the Fourier spectrum of the input data. This provides a computational efficient and simple way to freely move between the equally valid signal representations in the time, the frequency, and the time-frequency domains. However, we show that weighting the amplitude $\mathrm{S}$-spectrum to isolate/enhance signals can lead, due to the newly weighted Fourier spectrum, to a reconstructed time series with energy appearing at undesired places. It is shown in our examples that the imposed time localization through the weighting does not directly translate to the time domain. As a consequence, one does not really take advantage of the signal localization and isolation in the S-spectrum.

Our approach avoids the time averaging and is based on the individual consideration of each local spectrum as function of time (window center time). Each of these spectra is back transformed directly to the instant time that equals the window center time. This alternative strategy by-passes the need to average the local spectra and the signal localization in time translates directly to the filtered time series.

In fact, we consider the back transform as part of the filter since it may depend on the manipulation, its purpose, and the data at hand which strategy one should prefer. For 
our purposes the alternative back transform increases the quality of the filter outputs. There are less noise and spurious signals, and the waveforms seem to be less distorted. In any case, the results must be interpreted with care and with respect to the applied settings. Furthermore, a complete reconstruction of the waveforms should not be expected in the presence of large noise since corrupted signal components will also be down-weighted.

It would seem from the examples given here [5] that our approach can be useful to other applications based on the different weight functions. In our examples we employ the degree of polarization to isolate polarized signals. The degree of polarization reduces to a real number weighting such as other functionals based on other physical principals to attenuate noise in seismic data, in medical magnetic resonance images, and other applications based on a similar recipe. Whether the differences are large and justify a change of strategy depends on the data and weight.

Concerning the dop we would like to add that the polarization is subject to noise and that noise corrupted signals (noise interference in time and frequency) might not be detected by the approach. Furthermore, noise can be polarized, for instance if noise amplitudes are larger on one component than on the others and may not be suppressed. For densely spaced data such as seismic record sections, the isolated noise can be attenuated by lateral averaging of the dop ([4]). This improves considerably the signal detection through polarization. Noise suppression through averaging the degree of polarization is an alternative to average waveforms or covariance matrices. It has the advantage that one does not attenuate polarized signals with laterally changing waveforms which may happen in the transmission to postcritical reflections or due to focusing by heterogeneities.

\section{Acknowledgments}

We acknowledge the reviewers for their careful reading. Carine Simon is financed through a joint project between the Technical University of Catalonia (UPC) and the Marine Technology Unit (UTM). We are in debt to Antoni Manuel (UPC) and Juanjo Dañobeita (UTM) for their confidence, helpfulness and support. Martin Schimmel is funded through a Ramon and Cajal Fellowship.

\section{References}

[1] R. G. Stockwell, L. Mansinha and R. P. Lowe, Localization of the complex spectrum: The S transform, IEEE Trans. Signal Process., 44, 998-1001, 1996.

[2] P. D. McFadden, J. G. Cook, and L. M. Forster, Decomposition of gear vibration signals by the generalized $S$ transform, Mechanical Systems and Signal Processing 13, 691-707, 1999.

[3] C. R. Pinnegar and L. Mansinha. The S-transform with windows of arbitrary and varying shape, Geophysics 68, 381-385, 2003.

[4] M. Schimmel and J. Gallart, Degree of Polarization Filter for Frequency Dependent Signal Enhancement Through Noise Suppression, Bull. Seism. Soc. Am., 94, 1016-1035, 2004.

[5] M. Schimmel and J. Gallart, The inverse S-Transform in filters with time-frequency localization, IEEE Trans. Signal Process., in press, 2005. 